\title{
CGUG: in silico proteome and genome parsing tool for the determination of "core" and unique genes in the analysis of genomes up to ca. I.9 Mb
} Padmanabhan Mahadevan"1,2, John F King1,3 and Donald Seto*1

\begin{abstract}
Address: ${ }^{1}$ Department of Bioinformatics and Computational Biology, George Mason University, 10900 University Boulevard, MSN 5B3, Manassas, VA, 20110, USA, ${ }^{2}$ Current address: Department of Biological Sciences, Vanderbilt University, Nashville, TN 37235, USA and ${ }^{3}$ Current address: Kingdomain Corporation, 10305 Nantucket Court, Fairfax, VA 22032, USA

Email: Padmanabhan Mahadevan - padmahadevan@gmail.com; John F King - kingdomaincorp@gmail.com; Donald Seto* - dseto@gmu.edu

* Corresponding author
\end{abstract}

Published: 25 August 2009

BMC Research Notes 2009, 2:168 doi:10.1186/1756-0500-2-168
Received: 9 March 2009

Accepted: 25 August 2009

This article is available from: http://www.biomedcentral.com/1756-0500-2-168

(c) 2009 Seto et al; licensee BioMed Central Ltd.

This is an Open Access article distributed under the terms of the Creative Commons Attribution License (http://creativecommons.org/licenses/by/2.0), which permits unrestricted use, distribution, and reproduction in any medium, provided the original work is properly cited.

\begin{abstract}
Background: Viruses and small-genome bacteria $(\sim 2$ megabases and smaller) comprise a considerable population in the biosphere and are of interest to many researchers. These genomes are now sequenced at an unprecedented rate and require complementary computational tools to analyze. "CoreGenesUniqueGenes" (CGUG) is an in silico genome data mining tool that determines a "core" set of genes from two to five organisms with genomes in this size range. Core and unique genes may reflect similar niches and needs, and may be used in classifying organisms.

Findings: CGUG is available at http://binf.gmu.edu/geneorder.html as a web-based on-the-fly tool that performs iterative BLASTP analyses using a reference genome and up to four query genomes to provide a table of genes common to these genomes. The result is an in silico display of genomes and their proteomes, allowing for further analysis. CGUG can be used for "genome annotation by homology", as demonstrated with Chlamydophila and Francisella genomes.

Conclusion: CGUG is used to reanalyze the ICTV-based classifications of bacteriophages, to reconfirm long-standing relationships and to explore new classifications. These genomes have been problematic in the past, due largely to horizontal gene transfers. CGUG is validated as a tool for reannotating small genome bacteria using more up-to-date annotations by similarity or homology. These serve as an entry point for wet-bench experiments to confirm the functions of these "hypothetical" and "unknown" proteins.
\end{abstract}

\section{Background}

There is a tremendous increase in the number of genomes deposited in databases, with the data stream already a "data tsunami". The universal adoption of the "Next Generation" DNA sequencing technologies will also allow a parallel, expedited sequencing of smaller, but important and relevant, genomes such as from viruses and less than $2 \mathrm{Mb}$ bacterial genomes.

Software tools for taking advantage of these data need to be developed as well as maintained and upgraded for additional and more useful functions. In particular, the 
readily available and "user-friendly" computational tools, preferably platform-independent, are especially needed as many wet-bench researchers are interested in the informational content, the "biology," of the genomes rather than the computational aspects of these genomes.

CGUG is a modification and extension of a web-based tool, CoreGenes [1], which was limited to genomes of viruses (ca. $350 \mathrm{~kb}$ ), including chloroplasts and mitochondria. It now determines the "core" set of genes from a set of up to five bacteria with small genomes ( 2 Mb). Its usefulness in the small genomes community has attracted researchers with diverse interests and needs. In response to some of these interests and needs, the tool has been upgraded with the input of wet-bench researchers.

While bacteria with larger genomes, ca. $4+\mathrm{Mb}$, are of obvious importance, bacteria with genomes of smaller sizes are also of interest to the community; many of these are pathogens. Tools for data mining and analysis of the genomes and proteomes from these and other pathogens are important not only for understanding their basic biology, but also in the applications of these data for molecular surveillance and detection, including molecular diagnostics, as well as in drug design and discovery, including vaccine development.

For understanding the phylogeny of organisms, the determination of a set of common or "core" genes between a set of bacterial genomes provides insight into the particular and specific characteristics of those bacterial species and of their niches in the biosphere. Core genes are being used to reconstruct ancestral genomes [2], phylogenies [3] and organism classifications [4], and should provide insight into the common requirements of living in similar niches. The core set of genes has been used to explore the concept of the "pan-genome" of a bacterial species or a group of bacteria [5]. Essential genes comprising the minimal genome and the minimal life form, e.g., Mycoplasma genitalium [6] may be a subset of this core.

From a survey of the literature, there are relatively few tools for the determination of core genes from genomes. One example is CEGMA [7], which is used to annotate these in eukaryotic genomes. CEGMA is limited to the analysis of eukaryotic genomes. It is neither web-based nor functional across platforms, and must be downloaded and installed. Other tools have similar limitations or are confined to precomputed sets of genomes, or are no longer accessible/supported.

CGUG is a user-friendly "on-the-fly" web-based tool that determines, parses, analyzes and outputs a set of core genes from a set of two to five small bacterial genomes. As a validation of this tool, applications for analyzing
Chlamydophila and Francisella genomes are presented, including reannotation, especially 'hypothetical proteins', illustrating the comparisons of newly-determined genomes with the analysis with older, less well-annotated genomes; that is, to align and to identify similar and also putatively similar proteins, previously noted as "unknown" and "hypothetical" entries. The current and future versions of this tool are available at http:// binf.gmu.edu/geneorder.html.

In bacteriophage research, to complement the current classification criteria of the International Committee on the Taxonomy of Viruses (ICTV) [8] and to understand them better, a proteome tree analysis based on a BLASTP algorithm has been constructed earlier [9]. CGUG provides another independent in situ proteome analysis approach that incorporates suggestions by several ICTV members working on bacteriophages [4], noting that while these genomes contain horizontal transfers that have made understanding bacteriophage classification very difficult [4], a proteome-based approach can help to unravel and to understand their classifications [4].

\section{Implementation \\ Algorithm}

The algorithm is based on the GeneOrder algorithm to determine gene order and synteny [10]. GenBank accession numbers are inputted to select data files. These are extracted from GenBank and an iterative protein similarity analysis is performed for each protein from the query genome against the reference genome protein database using BLASTP from WU-BLAST.

\section{Limitations}

Currently, CGUG is limited to the analysis of small bacterial genomes (up to $2 \mathrm{Mb}$ ). Furthermore, it is limited to the analysis of five genomes at a time. Both limitations are due to the computational power and allocated memory of our server, which frequently comes under heavy user load; we hope to migrate this tool to a more powerful server. But for now, this tool is limited by computational resources (i.e., hardware) that restrict the size and number of genomes that can be processed. However, during our test runs, $4 \mathrm{Mb}$ genomes can be processed successfully. The caveat is that there is a significantly longer processing time ( $>1 \mathrm{hr}$; there is a queuing e-mail return option). Despite these limitations, CGUG is a valuable tool for biologists and this has been illustrated by its use in the classification of bacteriophages [4].

\section{Validation}

Chlamydophila analysis of core genes; annotation application Chlamydophila (1 Mb "small" genomes) are interesting because some are responsible for causing diseases in humans and other mammals: C. pneumoniae is a respira- 
tory pathogen that causes community-acquired pneumonia and bronchitis in humans [11]; C. felis causes conjunctivitis and upper respiratory tract disease in cats [12]; C. abortus causes abortions in ruminants such as sheep and goats [13]; and C. caviae causes conjunctivitis in guinea pigs [14]. Comparative genomics may provide insights into their biology as well as pathogenicity.

As an example of the reannotation application, Chlamydophila genomes, Table 1, are analyzed for their core genes, yielding a set of 839 related proteins, with a stringency or threshold range setting of "75" (default). A visual inspection of this output reveals many hypothetical proteins across the genomes. By looking at a specific row of putatively related genes, a hypothetical protein in one genome can be identified or annotated by comparison with annotated proteins noted in the other genomes. Figure 1 displays proteins annotated as O-sialoglycoprotein endopeptidase in C. pneumoniae J138 and in C. felis Fe/C56. The putatively related proteins in the same row are annotated as hypotheticals for C. abortus S26/3, C. pneumoniae AR39 and C. caviae GPIC. These must be analyzed further, as demonstrated in Figure 2 where CLUSTALWbased multiple sequence alignment (MSA) is presented. The extensive conserved residues and alignment suggest that the hypothetical proteins are likely O-sialoglycoprotein endopeptidases as well. Percent identities between the annotated proteins and the hypothetical proteins are relatively high, being $67 \%$ or greater, again, strongly suggests that these hypotheticals are O-sialoglycoprotein endopeptidases.

Another example is the annotation of a phosphohydrolase in C. pneumoniae J138 and in C. felis Fe/C-56; putatively related proteins are annotated as hypotheticals in

Table I: Accession numbers and sizes of five analyzed Chlamydophila genomes

\begin{tabular}{lll}
\hline Genome & Accession \# & Size (Mb) \\
\hline Chlamydophila pneumoniae JI38 & $\underline{N C ~ 002491}$ & 1.23 \\
\hline Chlamydophila felis Fe/C-56 & $\underline{N C ~ 007899}$ & 1.17 \\
\hline Chlamydophila abortus S26/3 & $\underline{N C ~ 004552}$ & 1.14 \\
\hline Chlamydophila pneumoniae AR39 & $\underline{N C ~ 002179}$ & 1.23 \\
\hline Chlamydophila caviae GPIC & $\underline{N C ~ 003361}$ & 1.17 \\
\hline Francisella tularensis SCHU S4 & $\underline{N C ~ 006570}$ & 1.89 \\
\hline Francisella tularensis holarctica & $\underline{N C ~ 009749}$ & 1.89 \\
\hline Francisella tularensis mediasiatica & $\underline{N C ~ 010677}$ & 1.89 \\
\hline
\end{tabular}

other genomes, Figure 3. Percent identities between the annotated proteins and the hypothetical proteins are $63 \%$ or greater, suggesting a similar function. Further analyses must be performed to confirm this; that is, the ultimate assignments of function lie in wet-bench experiments as annotation by homology and similarity can only suggest function.

Genome annotation and methods for annotation have lagged behind the DNA sequencing technology, in part, due to the vast unknown of the biology and coding potential of organisms. Genomes that have been sequenced more recently take full advantage of newly accumulated knowledge, and therefore are annotated more completely and, presumably, with less error. For the non-computational biologist who is interested in the biology of related organisms, inspection and alignments of genomes annotated from different time periods may be problematic. CGUG allows older genomes to be matched with related and recently sequenced genomes.

\section{Application to the larger Francisella genomes}

Francisella genomes are larger, at approximately $1.89 \mathrm{Mb}$. Important pathogens are among them, e.g., F. tularensis causes tularaemia [15]. Three genomes, Table 1, are analyzed to determine their "core" set of proteins and to note the reannotation function of CGUG. These organisms share 1229 core proteins. Figure 4 shows the partial output of the core proteins table, revealing a hypothetical protein in Francisella tularensis SCHU S4 (published 2004). Annotated counterparts in the recently sequenced Francisella tularensis holarctica and Francisella tularensis mediasiatica FSC147 (2007) show this as a major facilitator transporter and drug:H+ antiporter-1, respectively (Figure 5). Percent identities between the hypothetical protein and these two annotations are $99.2 \%$ and $99.7 \%$, strongly suggesting that the hypothetical protein is a transporter protein, again subject to validation by wetbench confirmation.

\section{Bacteriophage classifications}

Bacteriophages have been intensely studied in the laboratory, and their classifications have been debated and defined under current ICTV criteria, which include physical, clinical, biochemical and molecular data. Recently, several bacteriophage researchers have undertaken a reevaluation of the bacteriophages given the availability of genome data and the in situ proteome data. This data analysis included parsing the numbers of shared similar and orthologous proteins, using both CoreGenes and CoreExtractor.vbs [4]. The majority of the accepted relationships and ICTV classifications have been re-confirmed for the Podoviridae, although several new insights appeared. One example, three established genera within the T7-related bacteriophages are reconfirmed, along with 


\section{Figure I}

A row of output from CGUG showing related proteins from five Chlamydophila genomes. The annotated $O$ sialoglycoprotein endopeptidase in C. pneumoniae JI38 and C. felis Fe/C-56, respectively, are noted to have identity to counterparts noted in three Chlamydophila genomes. These additional columns display the equivalent and presumably related proteins which have been annotated originally as "hypothetical" in $C$. abortus, $C$. pneumoniae AR39 and C. caviae GPIC. This provides a lead for additional bioinformatic analyses and wet-bench investigations.

five putative novel genera. These proteome-inspired insights offer a refinement to the ICTV phage classification and provide a straightforward algorithm for the classification of new phage based on their genome and proteome [4]. The entire set of bacteriophages is being re-examined, beginning with the Podoviridae, above, and continuing with the Myoviridae, with plans for Siphoviridae and the rest.
As an example of CGUG analysis, bacteriophages from several genera of the Microviridae are analyzed in order to verify their current classification. These include Microvirus, Chlamydiamicrovirus, Bdellomicrovirus and Spiromicrovirus (Table 2). The first sequenced phage of each genus is used as the reference genome and is analyzed against the other members for shared similar proteins. A $40 \%$ cutoff for shared similar proteins is used for inclu-
C.pneumoniae_J138

C. felis_Fe/C-56

C. abortūs $526 / 3$

C.pneumoniae_AR39

C. caviae_GPI $\overline{\mathrm{C}}$

C.pneumoniae J138

C.felis_Fe/C-56

C.abortūs_s $26 / 3$

c. pneumoniae AR39

C.caviae_GPIC

C.pneumoniae_J138

C. felis $\mathrm{Fe} / \mathrm{C}-56$

C. abortūs_s $26 / 3$

C.pneumoniae_AR39

C.caviae_GPIC

C. pneumoniae J138

C.felis_Fe/C-56

C.abortús_s $26 / 3$

C. pneumoniae AR39

C.caviae_GPI $\bar{C}$

\begin{abstract}
MYFYKYVIIDTSGYYPFLACVDNQQVLEHWSLPVGPDLGIVLEFLFKSKNLSFQGVAVAL 60 MHFHRYVIIDTSGYQPFLAYVDHQKVLKHWQLPVGPDQGVVLEFIFKNSFLCFQGIGVAA 60 MYFHRYVIIDTSGYQPFLAYVDHQKVLKQWDLPVGPDQGLVLEFIFKNSSLSFQGIGVAV 60 MYFYKYVIIDTSGYYPELACVDNQQVLEHWSLPVGPDLGIVLEFLFKSKNLSFQGVAVAL 60 MHFHRYVIIDTSGYQPFLAYVDHQRVLKRWSLPVGPDQGLVLEFIFKNSGLCFQGIGVAA 60

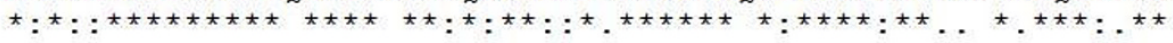

GPGNFSATRIGISFAQGLAMAKNVPLIGYSSLEGYLLSKDEKKALMLPLGKRGGVLTLSS 120 GPGNFSATRVGLSFAQGLALSKKVPMIGYSSLEGYLTPKDEGKALMLPLGKKGGVLTLSS 120 GPGNFSATRVGLSFAQGLALSRKVPMIGYSSLEGYLTPKDKGKALMLPLGKKGGVLTLSS 120 GPGNFSATRIGISFAQGLAMAKNVPLLGYSSLEGYLLSKDEKKALMLPLGKRGGVLTLSS 120 GPGNFSATRVGLSFAQGLALSRKVPMIGYSSLEGYLTPKDKGKALMLPLGKKGGVVTLSS 120

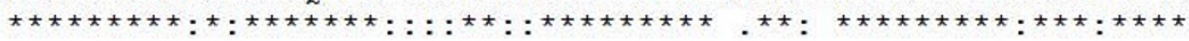

EIPEEGLNEKRRGVGPGALLSYEEASDYCVAHGYYHVISPNPQLFASSESDKITVEEVAP 180 DLSEDGFICEKNGVGPGILLPYGEASEYCLANNYYHVISPNPQLFIDSFSKKIRIEKVAP 180 DLSEDGEIHEKNGVGPGILLPYGEASKYCVANNCYHVISPNPELFIESESNRIRIEKAAP 180 EIPEEGLNEKRRGVGPGALISYEEASDYCVAHGYYHVISPNPQLFASSFSDKITVEEVAP 180 DLTEEGFIYEKNGVGPGILLPYEEASEYCLANHCYHVVSPNPQLFTDRFSNKIYIEETAP 180

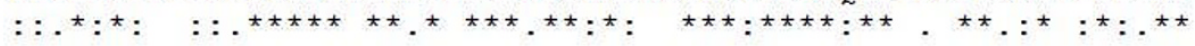

SVEQIRRHVISQFMFVEYDKQLSPDYRSYSCIF 213 SINCIRRHVVSQLMPLECGRQLTPDYRSCSCFF 213 SVDHIRRNVVSQLMILECSQQLTPDYRSCSCFF 213 SVEQIRRHVISQFMFVEYDKQLSPDYRSYSCIF 213 SIDHIRRNVVSQLMLLECSQQLTPDYRSCSCFF 213

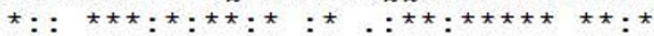

\section{Figure 2}

Multiple sequence alignment of five proteins from Chlamydophila genomes. The C. pneumoniae JI38 and C. felis Fe/C56 proteins displayed are annotated as O-sialoglycoprotein endopeptidase. CGUG analysis reveals counterpart proteins from C. abortus S26/3, C. pneumoniae AR39 and C. caviae GPIC that are annotated currently as "hypothetical proteins." As an example of additional bioinformatic analysis suggested by CGUG results, these counterparts are aligned to determine their identity to O-sialoglycoprotein endopeptidase. Conserved residues are indicated by asterisks. Colons indicate conserved substitutions, based on amino acid physico-chemical properties. Dots indicate semi-conserved substitutions. 


\begin{tabular}{l|l|l|l|l}
\hline GI:15836110 & GI: 89898651 \\
phosphohydrolase phosphohydrolase & GI: 62184806 & GI:16752460 & GI:29839930 \\
hyptical protein & hypothetical protein & hypothetical protein
\end{tabular}

\section{Figure 3}

Output of a row from CGUG showing phosphohydrolase-related proteins from five Chlamydophila genomes. The first two columns display an annotated phosphohydrolase protein in C. pneumoniae JI38 and C. felis Fe/C-56, respectively. The other three columns show related proteins from the CGUG result, annotated in the genome records as "hypothetical" for $C$. abortus, $C$. pneumoniae AR39 and C. caviae GPIC. This provides a lead for additional bioinformatic analyses and wet-bench investigations.

sion of a phage in a particular genus. This cutoff criterion has been used to verify the current classification of phages of the Podoviridae and to define novel genera as well, and also has been discussed in detail [4].

Using CGUG, Chlamydia phage 2 and Chlamydia phage $\varphi$ CPG1 share $50 \%$ similar proteins with Chlamydia phage 1. Chlamydia pneumoniae phage CPAR39 shares $42 \%$ similar proteins with Chlamydia phage 1 . These values are above the shared protein cutoff of $40 \%$ and verify the current ICTV classification in the Chlamydiamicrovirus genus. Proteins unique to Chlamydia phage 1, with respect to the other phages, include several hypothetical proteins and proteins annotated as "structural proteins". Table 3 shows the percent identities and BLAST E-values between the shared proteins of Chlamydia phage 1 and Chlamydia phage $\varphi$ CPG1. Even though many of the percent identities are not very high, several of the E-values suggest a signifi-

\begin{tabular}{|c|c|c|}
\hline $\begin{array}{l}\text { Francisella tularensis subsp. tularensis } \\
\text { SCHU S4 }\end{array}$ & Francisella tularensis subsp. holarctica FTNF002-00 & $\begin{array}{l}\text { Francisella tularensis subsp. mediasiatica } \\
\text { FSC147 }\end{array}$ \\
\hline $\begin{array}{l}\text { GI:56707188 } \\
\text { PRODUCT:chromosomal replication } \\
\text { initiator protein dnaA }\end{array}$ & $\begin{array}{l}\text { GI: } 169656467 \\
\text { PRODUCT:chromosomal replication initiator protein }\end{array}$ & $\begin{array}{l}\text { GI: } 187930914 \\
\text { PRODUCT:chromosomal replication initiator } \\
\text { protein DnaA }\end{array}$ \\
\hline $\begin{array}{l}\text { GI:56707189 } \\
\text { PRODUCT:DNA polymerase III, beta chain }\end{array}$ & $\begin{array}{l}\text { GI:156501372 } \\
\text { PRODUCT:DNA polymerase III, beta subunit }\end{array}$ & $\begin{array}{l}\text { GI: } 187930915 \\
\text { PRODUCT:DNA polymerase III, beta subunit }\end{array}$ \\
\hline $\begin{array}{l}\text { GI:56707191 } \\
\text { PRODUCT:MFS superfamily proline/ } \\
\text { betaine transporter }\end{array}$ & $\begin{array}{l}\text { GI: } 156501394 \\
\text { PRODUCT:major facilitator transporter }\end{array}$ & $\begin{array}{l}\text { GI: } 187932290 \\
\text { PRODUCT:metabolite:H+ symporter (MHS) } \\
\text { family protein }\end{array}$ \\
\hline $\begin{array}{l}\text { GI:56707192 } \\
\text { PRODUCT:aspartyl-tRNA synthetase }\end{array}$ & $\begin{array}{l}\text { GI:156501393 } \\
\text { PRODUCT:aspartyl-tRNA synthetase }\end{array}$ & $\begin{array}{l}\text { GI:187932291 } \\
\text { PRODUCT:aspartyl-tRNA synthetase }\end{array}$ \\
\hline $\begin{array}{l}\text { GI:56707195 } \\
\text { PRODUCT:adenylosuccinate lyase }\end{array}$ & $\begin{array}{l}\text { GI: } 156503322 \\
\text { PRODUCT:adenylosuccinate lyase }\end{array}$ & $\begin{array}{l}\text { GI:187930974 } \\
\text { PRODUCT:adenylosuccinate lyase }\end{array}$ \\
\hline $\begin{array}{l}\text { GI:56707196 } \\
\text { PRODUCT:hypothetical protein }\end{array}$ & $\begin{array}{l}\text { GI: } 156503321 \\
\text { PRODUCT:hypothetical protein }\end{array}$ & $\begin{array}{l}\text { GI: } 187930975 \\
\text { PRODUCT:hypothetical protein }\end{array}$ \\
\hline $\begin{array}{l}\text { GI:56707199 } \\
\text { PRODUCT:Glu-tRNAGln amidotransferase } \\
\text { C subunit }\end{array}$ & $\begin{array}{l}\text { GI: } 156503316 \\
\text { PRODUCT:Glu-tRNAGln amidotransferase C subunit }\end{array}$ & $\begin{array}{l}\text { GI: } 187930978 \\
\text { PRODUCT:glutamyl-tRNA(Gln) } \\
\text { amidotransferase, C subunit }\end{array}$ \\
\hline $\begin{array}{l}\text { GI:56707200 } \\
\text { PRODUCT:glutamyl-tRNA(Gln) } \\
\text { amidotransferase subunit A }\end{array}$ & $\begin{array}{l}\text { GI: } 156503315 \\
\text { PRODUCT:aspartyl/glutamyl-tRNA(Asn/Gln) amidotransferase, } \\
\text { subunit A }\end{array}$ & $\begin{array}{l}\text { GI:187930979 } \\
\text { PRODUCT:glutamyl-tRNA(Gln)/aspartyl- } \\
\text { tRNA(Asn) amidotransferase, A subunit }\end{array}$ \\
\hline $\begin{array}{l}\text { GI:56707201 } \\
\text { PRODUCT:aspartyl/glutamyl-tRNA } \\
\text { amidotransferase subunit B }\end{array}$ & $\begin{array}{l}\text { GI:169656772 } \\
\text { PRODUCT:aspartyl/glutamyl-tRNA(Asn/Gln) amidotransferase, } \\
\text { subunit B }\end{array}$ & $\begin{array}{l}\text { GI: } 187930980 \\
\text { PRODUCT:glutamyl-tRNA(Gln)/aspartyl- } \\
\text { tRNA(Asn) amidotransferase, B subunit }\end{array}$ \\
\hline
\end{tabular}

\section{Figure 4}

Output of "core" set of proteins from three Francisella genomes. Partial output of the "core" set of proteins from Francisella tularensis SCHU S4, Francisella tularnensis holarctica and Francisella tularensis mediasiatica are presented as an example of the core set of genes amongst these organisms. Each is linked to their GenBank record and may be retrieved for additional bioinformatic analyses. 


\begin{tabular}{|c|c|}
\hline Gl:1:60072066 & Gi:1:16603309 \\
\hline
\end{tabular}

\section{Figure 5}

Row of output from three Francisella genomes. Counterpart proteins from Francisella genomes are displayed: the first column corresponds to Francisella tularensis SCHU S4; the second column corresponds to Francisella tularnensis holarctica; and the third column corresponds to Francisella tularensis mediasiatica. As noted in the text, the counterpart annotations provide a clue as to the function of the "hypothetical" protein, subject to additional bioinformatic analyses and wet-bench investigations.

cance of alignments and relationships. Caveat: Need wetbench experiments to confirm the functional properties.

Bdellovibrio phage $\varphi \mathrm{MH} 2 \mathrm{~K}$, which belongs to the Bdellomicrovirus genus, shares significantly less than $40 \%$ similar proteins with the phages of the Microvirus genus. Specifically, it shares no similar proteins with $\varphi \mathrm{X} 174, \mathrm{G} 4$ and $\varphi \mathrm{K}$. It only shares one protein with $\alpha 3$ and S13. Bdellovibrio phage $\varphi \mathrm{MH} 2 \mathrm{~K}$ also shares less than $40 \%$ similar proteins with a phage of the Spiromicrovirus genus, Spiroplasma phage 4 . These results justify the current separation of

Table 2: Accession numbers and sizes of analyzed bacteriophage genomes

\begin{tabular}{|c|c|c|}
\hline Genome & Accession \# & Size (bp) \\
\hline Enterobacteria phage $\alpha 3$ & $\underline{N C 001330}$ & 6087 \\
\hline Enterobacteria phage G4 & NC 001420 & 5577 \\
\hline Enterobacteria phage $\varphi \times 174$ & NC 001422 & 5386 \\
\hline Enterobacteria phage $\mathrm{SI} 3$ & $\underline{A F 27475 I}$ & 5386 \\
\hline Enterobacteria phage $\varphi \mathrm{K}$ & $\times 60323$ & 6089 \\
\hline Chlamydia phage I & NC 00174I & 4877 \\
\hline Chlamydia phage 2 & NC 002194 & 4563 \\
\hline Chlamydia pneumoniae phage CPAR39 & NC 002180 & 4532 \\
\hline Chlamydia phage $\varphi$ CPGI & NC 001998 & 4529 \\
\hline Bdellovibrio phage $\varphi \mathrm{MH} 2 \mathrm{~K}$ & NC 002643 & 4594 \\
\hline Spiroplasma phage 4 & NC 003438 & 4421 \\
\hline Enterobacteria phage T7 & NC 001604 & 39,937 \\
\hline Enterobacteria phage P22 & NC 00237I & 41,724 \\
\hline Enterobacteria phage lambda & NC 001416 & 48,502 \\
\hline
\end{tabular}

Bdellovibrio phage $\varphi \mathrm{MH} 2 \mathrm{~K}$ from the Microvirus and Spiromicrovirus genera. In contrast, Bdellovibrio phage $\varphi \mathrm{MH} 2 \mathrm{~K}$ shares approximately $45 \%$ similar proteins with the phages of the Chlamydiamicrovirus genera. There are discussions on merging these two genera; these in silico proteome results from CGUG lend more support to this position.

\section{Continuing development}

Software development is an on-going process, both in terms of coding and hardware as well as research needs. CGUG is an example of this, being supported and updated in response to requests from researchers, e.g., reanalysis of all bacteriophages, and supported in regards to coding updates. A beta version (CGUG 3.1), at the same site, is an alternative and complementary upgrade that will continue to be improved. It provides a more robust user interface (UI) and aims to improve the user experience, including a time bar to monitor the run length. It provides for a better batch analysis, recommended especially for long running queries, such as for the $2 \mathrm{Mb}$ genomes, and in preparation for the much larger bacterial genomes in the future, $c a$. $>4 \mathrm{Mb}$. Algorithm enhancements are needed and planned, as the current implementation does not handle these long running queries robustly. The feature list below summarizes anticipated current and continuing work:

- Improve user interface (UI)

- Show a dynamic status indicator of query progress

- Allow user to elect to receive results via email at any time

- Review implementation of algorithm for performance

- Add persistence (e.g., database) of queries and results by user 
Table 3: Percent identities and E-values between shared proteins of Chlamydia phage I and Chlamydia phage $\varphi$ CPG I

\begin{tabular}{lllc}
\hline Chlamydia phage I & Chlamydia phage $\varphi$ CPG I & \% identity & E-value \\
\hline VPI & hypothetical protein & 49.0 & e-160 \\
\hline VP2 & capsid protein VP2-related protein & 24.6 & $2 \mathrm{e}-25$ \\
\hline VP3 & capsid protein VP3 & 25.3 & $3 \mathrm{e}-13$ \\
\hline hypothetical protein & nonstructural protein & 60.0 & $2 \mathrm{e}-7 \dagger$ \\
\hline hypothetical protein & hypothetical protein & 18.9 & $7 \mathrm{e}-21$ \\
\hline nonstructural protein & nonstructural protein & 30.2 & $3 \mathrm{e}-8$
\end{tabular}

The " $\dagger$ " indicates that the E-value was obtained with the low complexity filter in b/2seq turned off. This was done because the proteins are short ( 36 amino acids).

CoreGenes was originally designed for a nominal use case of a single query submission with the user waiting for the results page to be returned (synchronous mode); 3.1 now provides a better Batch Analysis mode option where the user provides their email address for subsequent delivery of results. The site is redesigned using Google Web Toolkit (GWT) technology, which is ideal for the requirements of a potentially long running response in a web-based application. GWT is based on Asynchronous JavaScript and XML (AJAX), which allows for a much more robust and interactive user experience in a browser-based application.

In this beta version (3.1) of CGUG, when the user submits a query, the web page indicates that the query has begun executing and will present the user with a query status indicator (e.g., a "progress bar"), with a message log. Once a query is submitted and has begun executing, the approximate number of iterations that will be required to complete the computation will be known. With minor modifications, the Java program that executes the query on the server will track the iterations completed and report back to the user progress via "call back" mechanisms that are easily implemented with GWT. Based on this, a rough "percent complete" indicator is displayed and will be updated continuously via a client side timer executing in JavaScript in the browser. Thus, the progress indicator will update automatically with no action required by the user, allowing for real-time updating.

\section{Conclusion}

CGUG is an in silico genome and proteome data mining tool that is useful in the analysis of core genes from smallgenome bacteria ( $\sim 2 \mathrm{Mb})$, and in the putative assignments and suggestions of function for genes previously annotated as unknown or hypothetical, taking advantage of the new genomes and annotations as well as the growing databases for protein function assignment.

Another dimension of CGUG is realized in the reanalysis and verification of the current classifications of organisms, for example in the reanalysis and new insights of bacteriophages.

\section{Availability and requirements Project name: CGUG}

Project home page: http://binf.gmu.edu:8080/ CoreGenes3.0 and general splash page, http:// binf.gmu.edu/geneorder.html (including version 3.1)

Operating system(s): Platform independent web-based

Programming language: Java, XML

Any restrictions to use by non-academics: License required for commercial usage

\section{Competing interests}

The authors declare that they have no competing interests.

\section{Authors' contributions}

PM implemented the software and performed the analyses. JFK provided additional ideas and coding. DS conceived the project. PM, JFK and DS wrote the manuscript. All authors read and approved the final manuscript.

\section{Acknowledgements}

We acknowledge gratefully Drs. Andrew Kropinski and Rob Lavigne for suggestions of features, and for their collaboration and validation in applying CGUG to their studies of bacteriophages. We thank Chris Ryan for providing systems administration and server support and Jason Seto for providing support and a critical reading and editorial comments. We are 
grateful to the Apache Software Foundation (Tomcat), the Regents of the University of California (Ptolemy Plot) and Google (Google Web Toolkit) for allowing community access to their software as open resources.

\section{References}

I. Zafar N, Mazumder R, Seto D: CoreGenes: a computational tool for identifying and cataloging "core" genes in a set of small genomes. BMC bioinformatics 2002, 3:12.

2. Koonin EV: Comparative genomics, minimal gene-sets and the last universal common ancestor. Nature reviews 2003, I(2): $127-136$

3. Lerat $E$, Daubin $V$, Moran NA: From gene trees to organismal phylogeny in prokaryotes: the case of the gamma-Proteobacteria. PLoS biology 2003, I(I): EI 9.

4. Lavigne R, Seto D, Mahadevan P, Ackermann HW, Kropinski AM: Unifying classical and molecular taxonomic classification: analysis of the Podoviridae using BLASTP-based tools. Research in microbiology 2008, 159(5):406-4I4.

5. Tettelin H, Masignani V, Cieslewicz MJ, Donati C, Medini D, Ward NL, Angiuoli SV, Crabtree J, Jones AL, Durkin AS, et al:: Genome analysis of multiple pathogenic isolates of Streptococcus agalactiae: implications for the microbial "pan-genome". Proceedings of the National Academy of Sciences of the United States of America 2005, 102(39): I3950-13955.

6. Medini D, Donati C, Tettelin H, Masignani V, Rappuoli R: The microbial pan-genome. Current opinion in genetics \& development 2005, I 5(6):589-594.

7. Parra G, Bradnam K, Korf I: CEGMA: a pipeline to accurately annotate core genes in eukaryotic genomes. Bioinformatics (Oxford, England) 2007, 23(9): 106I-1067.

8. Fane B: Microviridae. In Virus taxonomy: classification and nomenclature of viruses: eighth report of the International Committee on Taxonomy of Viruses Edited by: Fauquet C, Mayo MA, Maniloff J, Desselberger U, Ball LA. San Diego; London: Elsevier Academic Press; 2005:288-299.

9. Rohwer F, Edwards R: The Phage Proteomic Tree: a genomebased taxonomy for phage. Journal of bacteriology 2002, 184(16):4529-4535

10. Mazumder R, Kolaskar A, Seto D: GeneOrder: comparing the order of genes in small genomes. Bioinformatics (Oxford, England) 200I, 17(2):162-166.

II. Hahn DL, Azenabor AA, Beatty WL, Byrne Gl: Chlamydia pneumoniae as a respiratory pathogen. Front Biosci 2002, 7:e66-76.

12. Cai Y, Fukushi H, Koyasu S, Kuroda E, Yamaguchi T, Hirai K: An etiological investigation of domestic cats with conjunctivitis and upper respiratory tract disease in Japan. The Journal of veterinary medical science/the Japanese Society of Veterinary Science 2002, 64(3):215-219.

13. Szeredi L, Janosi S, Tenk M, Tekes L, Bozso M, Deim Z, Molnar T: Epidemiological and pathological study on the causes of abortion in sheep and goats in Hungary (1998-2005). Acta veterinaria Hungarica 2006, 54(4):503-5I5.

14. Strik NI, Alleman AR, Wellehan JF: Conjunctival swab cytology from a guinea pig: it's elementary! Veterinary clinical pathology/ American Society for Veterinary Clinical Pathology 2005, 34(2):169-171.

15. Nigrovic LE, Wingerter SL: Tularemia. Infectious disease clinics of North America 2008, 22(3):489-504.

\section{Publish with Bio Med Central and every scientist can read your work free of charge}

"BioMed Central will be the most significant development for disseminating the results of biomedical research in our lifetime."

Sir Paul Nurse, Cancer Research UK

Your research papers will be:

- available free of charge to the entire biomedical community

- peer reviewed and published immediately upon acceptance

- cited in PubMed and archived on PubMed Central

- yours - you keep the copyright

Submit your manuscript here:

http://www.biomedcentral.com/info/publishing_adv.asp
BioMedcentral 